\title{
Brightness and image definition of pictures viewed from between the legs
}

\author{
Atsuki Higashiyama $\cdot$ Miyuki Toga
}

Published online: 9 November 2010

(C) Psychonomic Society, Inc. 2010

\begin{abstract}
It has been documented that if we see a scene from between our own legs, it appears brighter and more distinct. We determined factors that are critical to these changes and elucidated how they are related to visual disorder that is contingent on bending the head. In Experiment 1, upright scene pictures were viewed with the head inverted. In Experiments 2 and 3, the $180^{\circ}$ rotated versions of the same pictures were seen with the head inverted and upright, respectively. In Experiment 4, geometric patterns were seen with the head inverted. In Experiment 5, apparent depth and organization of scene pictures were judged under combinations of retinal-image and head orientations. The first results demonstrated that the scene pictures, when seen with the head inverted, had a $9.8 \%$ increase in brightness and a $6.8 \%$ increase in image definition as compared to normal upright viewing, but these changes did not arise for geometric patterns. Second, these changes were mainly ascribed to a proprioceptive change of the head. Third, by inverting only the head or the retinal image, visual structure of pictures was disturbed but the visual disorder did not always change brightness and image definition. These findings are discussed.
\end{abstract}

Keywords Head-inversion effects · Brightness · Image definition $\cdot$ Apparent depth $\cdot$ Scene perception

\footnotetext{
A. Higashiyama $\cdot$ M. Toga

Ritsumeikan University,

Kyoto, Japan

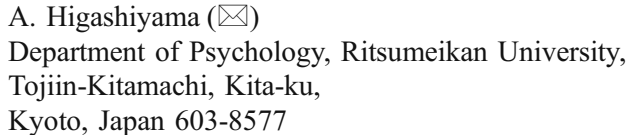

In this paper, it is assumed that, during a long period after birth, we learn ways of seeing size, distance, direction, and even color of objects. It is also assumed that these acquired visual skills are elaborated in the upright position of the head, so that the orientation of the head works as a frame of visual space. That is, the proprioceptive and tactile senses of the body are connected to prominent properties of perceived space. For example, even if the eyes are occluded, an upright person perceives the jaw to be directed toward the ground, the vertex to be directed toward the sky, and the sole of the foot to be in contact with the ground. By putting the head between the legs or by tilting it laterally, however, this connection between proprioception of the body and properties of visual space is greatly altered, and the usual way of seeing, on which we normally rely, does not work well.

A change in proprioception of the eyes, the head, or the body actually affects visual spatial perceptions including direction, size, and distance. Specifically, the visual vertical changes with a change in orientation of the head or the body, as known as the A-effect and the E-effect (Howard, 1982; Howard \& Templeton, 1966, for review). Apparent size (Ross \& Plug, 2002, for apparent size of the moon) and apparent distance (Galanter \& Galanter, 1973) also change as a function of direction of gaze and orientation of the headbody assembly, respectively. Higashiyama and Adachi (2006) recently demonstrated that an unaccustomed posture of bending the body definitely diminishes the degree of size constancy and foreshortens the apparent distance of a remote target.

This kind of connection between bodily proprioception and visual perception is, however, not very evident in all qualities of visual perception. In particular, it remains open how proprioception of the eyes, the head, or the body affects perceptions of color and lightness. One example 
where this connection is suggested is the head-inversion effect. When a landscape is viewed with the head inverted, by putting it under the arm or by putting it between the legs, it appears brighter and has better image definition than when viewed with the head upright (Cornish, 1935; Gibson, 1950; Ross, 1974; Sanford, 1898; von Helmholtz, 1910/1962). Brightness is generally referred to as a visual experience of a single color patch along the dark-dim-bright dimension, but we refer to brightness as a visual experience that is brought by the whole of a scene. Image definition may be referred to as a compound of clearness, sharpness, and vividness for neighboring color patches, but we refer to image definition as one that is brought about by the whole of a scene. We also use the adjective "distinct" instead of "definite" to describe image definition.

When a scene is viewed with the head upside down, what makes it appear brighter and more distinct? In this situation, several sensory factors change as compared to normal upright posture. First, the lower back muscles are stretched and the belly muscles are contracted (i.e., muscular imbalance); second, vestibular stimulation in the inner ears is disturbed (i.e., vestibular disturbance); third, the head is congested with blood (i.e., inner pressure by blood); finally, orientation of the retinal image is reversed from left to right and is also inverted from up to down, which is equivalent to the $180^{\circ}$ rotation of the visual field (i.e., retinal orientation).

Interestingly enough, very few studies have attempted to identify the sources that may produce the change in brightness or image definition in the head-inversion perception. This may be because many researchers, according to von Helmholtz's (1910/1962) remark, have believed that orientation of the retinal image is critical to the headinversion effects. He noted that with the head upside down, we are more sensitive to color differences, because color constancy in the inverted retinal image is diminished. Beck $(1969,1972)$ indicated that the orientation of a photographic picture with respect to an upright observer affects the perception of lightness and clearness. His observers were shown a picture of a young girl, first right side up and then inverted. The observers reported that (1) the chest of the child appeared to be a lighter gray when the picture was upright than when it was inverted, and (2) the area near the child's neck appeared much clearer when the picture was inverted than when it was upright. Beck (1972) interpreted these findings as meaning that when the picture is upright, we see the chest and face as single surfaces of a specific lightness, and variation of luminance is seen as shadow and highlights that model the surfaces; but when the picture is inverted, such relatedness of the areas changes so that the picture is seen as made up of separate areas and appears flatter.
Miyakawa (1949b), who was probably the first to measure the head-inversion effects psychophysically, compared the appearance of achromatic targets (i.e., a homogeneous gray disk and a line drawing of an elephant) between the upright and inverted heads. When these targets were viewed with the head upside down, they were perceived as film color and appeared brighter regardless of whether the targets were upright or inverted. He thus ascribed the head-inversion effects to a muscular or vestibular disturbance. Evidently, this result did not support Helmholtz's account, but his paper, written in Japanese, has not been familiar to Western researchers (see also Miyakawa, 1943, 1949a, in which he addressed problems of apparent distance in the between-leg situation).

With this argument in mind, in Experiments 1 to 3 of this paper, we attempted to identify sensory factors that affect the head-inversion perception. In Experiment 1, as basic measurements, participants viewed naturalistic pictures with the head between the legs and judged brightness or image definition of each picture. To invert only the head, in Experiment 2, participants viewed the $180^{\circ}$-rotated pictures with the head between the legs. In Experiment 3, participants viewed the rotated pictures with the head upright, which brought rotation of only the retinal image. If the outcomes of Experiment 2 resemble the basic measurements of Experiment 1, it is suggested that muscular, vestibular, or pressure disturbance is more influential than orientation of the retinal image. If the outcomes of Experiment 3 resemble the basic measurements, orientation of the retinal image would be more critical than muscular, vestibular, or pressure disturbance.

How can we explain the changes in brightness and image definition that are contingent on orientation of the head? One possible explanation is based on a change in apparent depth that may be associated with orientation of the head. von Helmholtz (1910/1962) stated that the green color of a lawn appears green regardless of whether it is at a near or far distance (i.e., color constancy), but with the head inverted, the landscape appears like a flat picture, and the green of the lawn appears green at a near distance and blue grey at a very far distance. Another explanation similar to this is based on the dissociation of colors from objects. Sanford (1898) stated that, in an upright position of the head, colors are attended to as signs of objects, but in their inverted positions, the association between color and object is loose and colors are seen more independently of objects. These explanations assume that a change in color and brightness in the head-inversion perception is caused by the visual disorder of a scene (i.e., reduction of depth and dissociation of colors from objects) that may follow a change in proprioception of the head or a change in orientation of the retinal image. We will call these explanations a visual disorder hypothesis. 
A possible alternative is that, when we see a scene from between the legs, a change in proprioception of the body directly arouses the color and brightness system. This explanation is based on the physiological fact that a hapticvisual interaction actually occurs at the posterior parietal cortex: Proprioceptive information from the somatosensory area in the postcentral gyrus is there mixed with visual information that is sent out through a dorsal pathway of visual processing (e.g., Goodale \& Milner, 2004). In this site, proprioceptive information may enhance brightness and image definition intermodally. We will call this a proprioception hypothesis.

These hypotheses were examined in Experiment 4 where the participants were exposed to geometric patterns that have neither intrinsic orientation nor pictorial cues to depth. Since such patterns appear flat, regardless of the orientation of the head, we do not predict the head-inversion effects from the visual disorder hypothesis, but predict them from the proprioception hypothesis.

The visual disorder hypothesis was also examined in Experiment 5, where participants viewed the upright or inverted naturalistic pictures with the head upright or inverted, and judged apparent depth and relatedness of parts in the pictures. The visual disorder hypothesis predicts that visual scenes are disorganized whenever brightness and image definition of pictures are facilitated as a consequence of a change in orientation of the head or of the retinal image. The proprioception hypothesis, however, does not predict the disorder of visual scenes.

\section{Method}

Participants The participants were 96 university students (40 males and 56 females; age range, 18-39 years; average age, 21.7 years). They were all paid for their participation.

Stimuli The standards were four matte-colored photographic pictures (a baby nestled in his father's arms, a river in mist, a garden in spring, and morning glories on the gate) as shown in Fig. 1. All pictures were natural scenes that had intrinsic orientations and pictorial cues to depth. Each standard was printed on paper by a Fujizerox-DocuPrint-C3540 printer. The three standards were $21 \mathrm{~cm}$ high $\times 29.5 \mathrm{~cm}$ wide and one was $29.5 \mathrm{~cm}$ high $\times 21 \mathrm{~cm}$ wide. The comparisons were 11 pictures that were made for each standard, differing from -25 to $+25 \%$ in $5 \%$ steps in brightness or contrast, as defined by Adobe Photoshop. The comparisons that differed in brightness were used for brightness judgments and the comparisons that differed in contrast were used for imagedefinition judgments. Each comparison was printed on paper by the same printer and its size was entirely the same as the standard size.
Calibration patterns To examine whether luminance of the printed pictures is proportional to Adobe's percent brightness and contrast, respectively, we made an additional pattern (Fig. 1) that was composed of 12 arbitrarily chosen color patches, which were arranged in three lines and four rows:

$\operatorname{red}_{(255,0,0)}, \operatorname{green}_{(0,255,0)}$, blue $_{(0,0,255)}, \operatorname{yellow}_{(255,255,0)}$

$\operatorname{cyan}_{(0,255,255)}, \operatorname{magenta}_{(255,0,255)}$, white $(255,255,255)$, black $_{(0,0,0)}$

$\operatorname{gray}_{(127,127,127)}$ darkred $_{(127,0,0)}$ darkyellow $_{(127,127,0)}$ darkblue $_{(0,0,127)}$

where the triple values in each parenthesis represent Adobe's (r, g, b). From this pattern, 11 calibration patterns that varied from -25 to $+25 \%$ in Adobe's per-cent brightness or contrast were made on a computer display and then printed in the same way as the scene pictures. Each calibration pattern was $21 \mathrm{~cm}$ high $\times 29.5 \mathrm{~cm}$ wide.

Colors of each printed calibration pattern were measured with a Minolta CS-100A luminance and chromaticity meter. Figure 2 shows the luminance of each colored patch against Adobe's percent brightness. Clearly, the luminance of any patch almost linearly increased as the percent brightness increased. We obtained, by a least-square criterion, a slope of luminance of each patch as a function of the percent brightness (see legends of Fig. 2). Although some patches (i.e., Gray and Dark Yellow) were steep in slope and others (i.e., Green and Blue) were shallow, all colors were positive in slope except for Black. This implies that, as the percent brightness increased, the patches in printed scene pictures, as a whole, increased in luminance. Figure 3 shows the chromaticity of each patch as a function of the per-cent brightness. No systematic or large change was found in the chromaticity.

Figure 4 shows the luminance of each patch as a function of Adobe's percent contrast. We also obtained a slope of luminance of each patch against the per-cent contrast (see legends of Fig. 4). White and Yellow were steep in slope, whereas others were very shallow (i.e., Cyan and Dark Yellow) or almost zero (e.g., Green and Black). This implies that, as the percent contrast increased, contrasts among patches were enhanced in printed scene pictures. Figure 5 shows the chromaticity of each patch as a function of the percent contrast. The chromaticity was almost constant, independently of change in percent contrast.

Design There were three experiments. In each experiment, 16 participants judged the brightness of pictures and a separate 16 participants judged their image definition. In the brightness or image-definition judgments, each participant undertook both experimental and control conditions. The experimental condition differed among the experiments: in Experiment 1, each participant observed the upright standard 


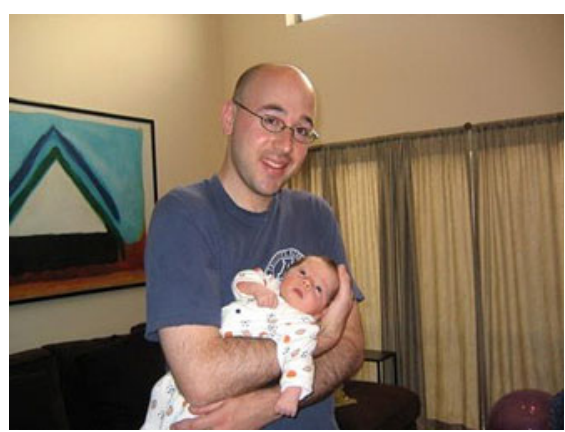

Baby

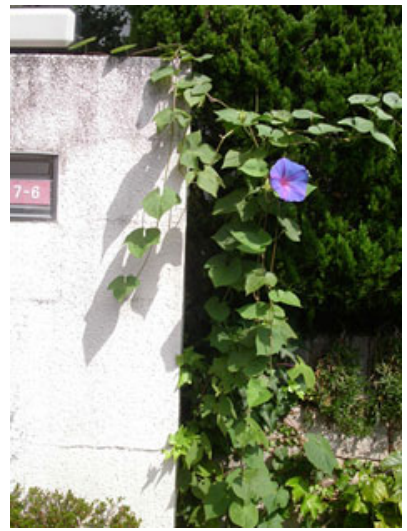

Morning glories

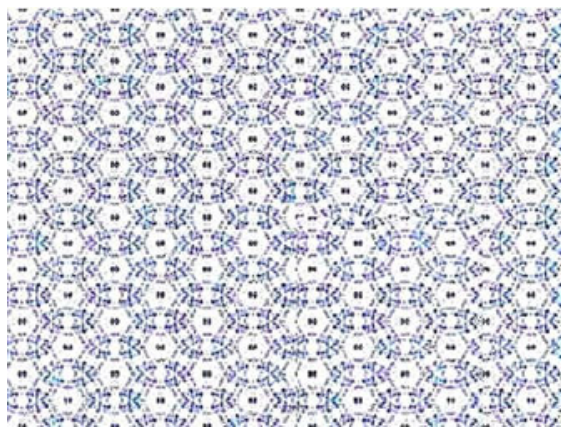

Circles

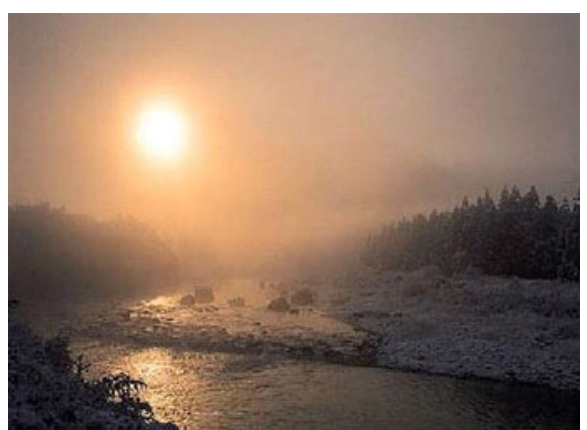

River

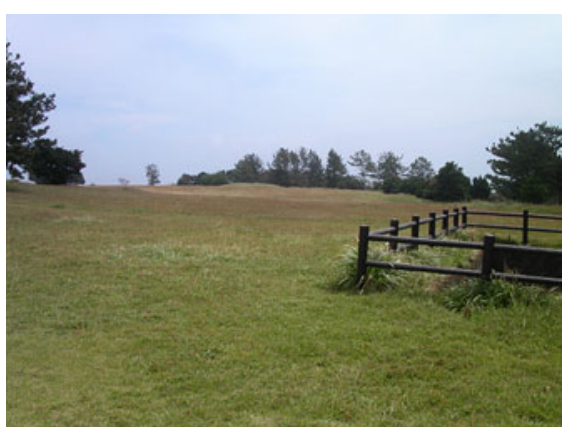

Garden

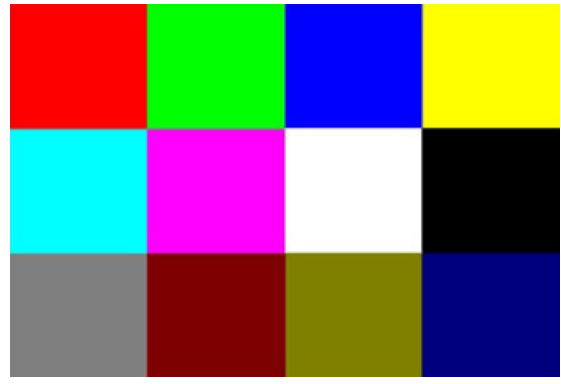

Calibration pattern

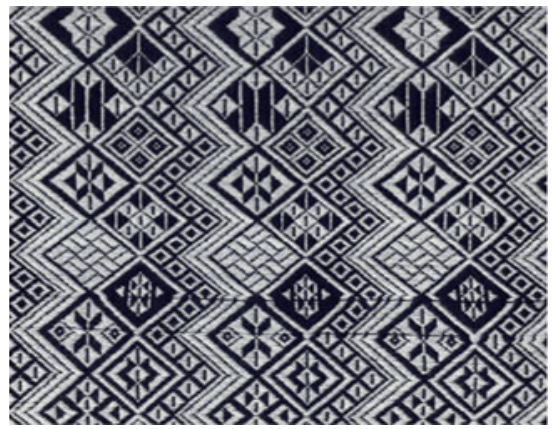

Squares

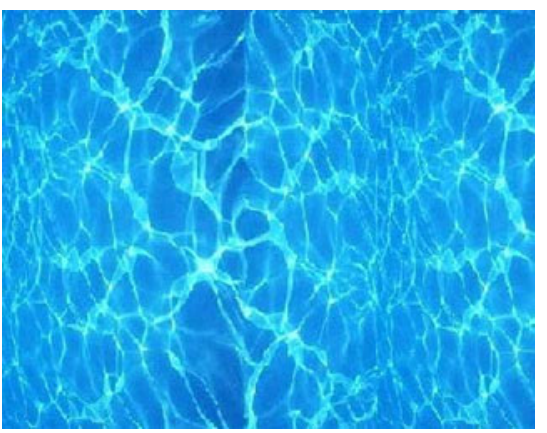

Waves

Fig. 1 The standard pictures: naturalistic scene pictures (i.e., Baby, River, Garden, and Morning Glories) used in Experiments 1 to 3 and geometric patterns (i.e., Circles, Squares, and Waves) used in Experiment 4

with the head between the legs and then judged the upright comparison with the head upright (i.e., inversion of both the retinal image and the head); in Experiment 2, each participant observed the upside-down standard with the head between the legs and then judged the upright comparison with the head upright (i.e., inversion of only the head); in Experiment 3, each participant observed the upside-down standard with the head upright and then judged the upright comparison with the head upright (i.e., inversion of only the retinal image). The control condition was the same in all experiments: each participant observed the upright standard with the head upright and then judged the upright comparison with the head upright (i.e., normal upright viewing).
Procedure The room used for the experiment had four white walls one of which had several windows. The room was illuminated with ordinary fluorescent lamps on the ceiling, and the floor near the windows was illuminated with natural light coming through the windows from the outside.

The standard was presented on a side wall and the comparison was presented on the wall opposite to the standard. The viewing distance of each picture was $1.2 \mathrm{~m}$. The height of the picture from the floor depended on stature of the participants and their muscular flexibility of the lower back: in the case of erect posture, the center of each picture was $140-170 \mathrm{~cm}$ above the floor, and in the case of bent posture, it was $10-30 \mathrm{~cm}$ above the floor. When 


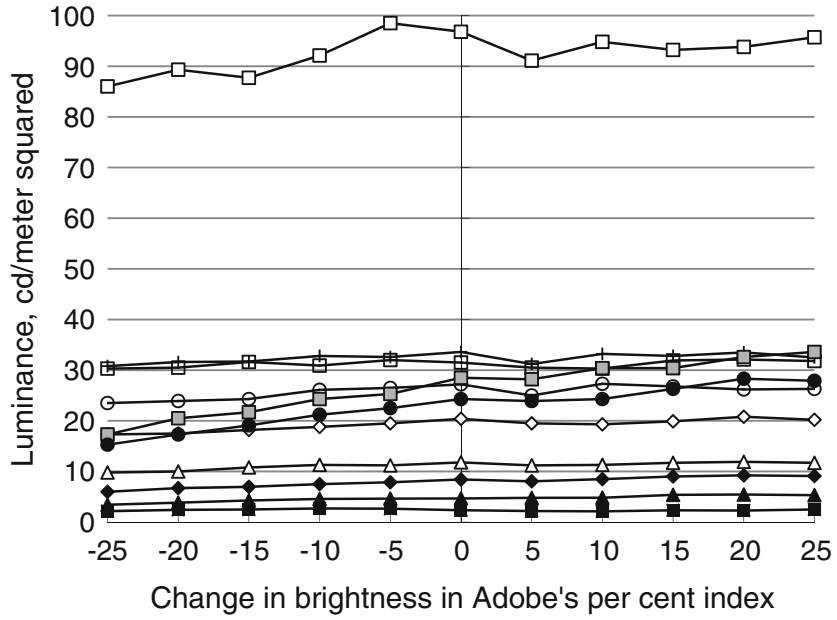

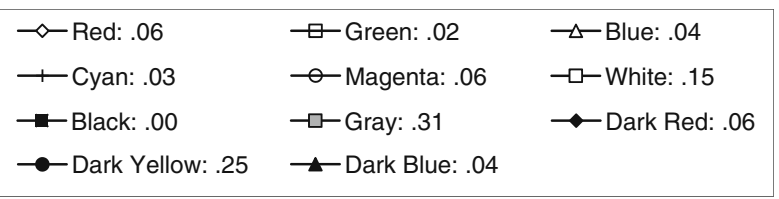

Fig. 2 Luminance of the calibration pattern as a function of Adobe's percent brightness. The slopes of least-square lines are indicated in the legends

the participants stood erect and observed a picture with the head upright, their chests were always directed toward the picture, whereas when the participants bent their bodies forward and observed a picture from between their legs, their backs were always turned to the picture.

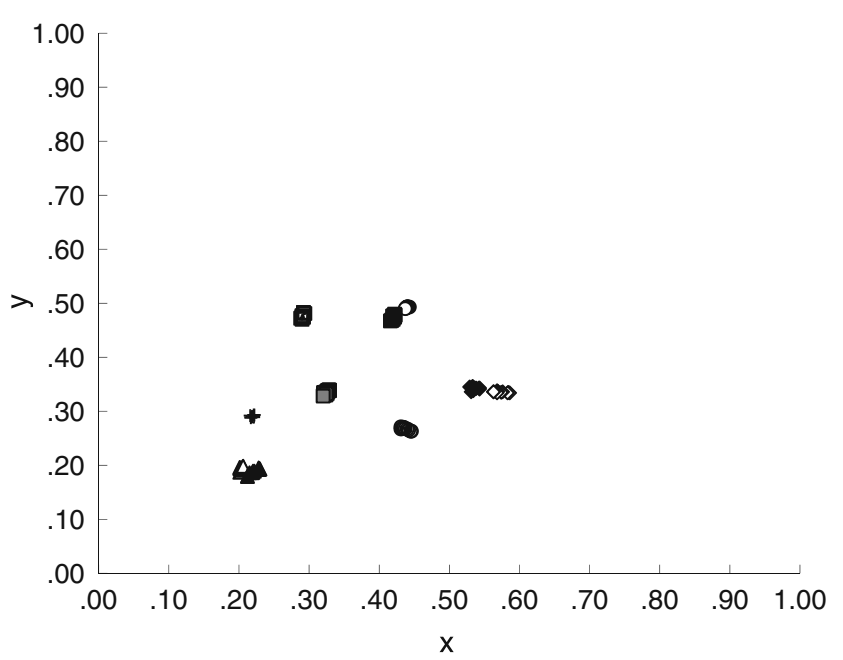

$$
\begin{aligned}
& \leadsto \text { Red (.57, .34) } \quad \square-\operatorname{Green}(.29, .48) \quad-\square \text { Blue(.20, .19) } \\
& \multimap \text { - Yellow(.44, .49) —C Cyan(.22, .29) - } \\
& \rightarrow-\text { White(.33, .34) } \rightarrow \text { Black(.32, .33) } \quad \rightarrow-\operatorname{Gray}(.32, .33) \\
& \longrightarrow \text { Dark Red(.54, .34) } \longrightarrow \text { Dark Yellow(.42, .47) } \longrightarrow \text { Dark Blue(.22, .19) }
\end{aligned}
$$

\begin{tabular}{|c|c|c|}
\hline$\prec$ Red: .03 & $\square$ Green: -.01 & $\triangle-$ Blue: .04 \\
\hline -ం-Yellow: .23 & —Cyan: .02 & ○-Magenta: .07 \\
\hline$\rightarrow \square-W h i t e: .29$ & $\rightarrow$-Black: .00 & $\rightarrow \square-G r a y: .06$ \\
\hline$\multimap$ Dark Red: .00 & $\multimap-D a r k$ Yellow: .04 & ——Dark Blue: .00 \\
\hline
\end{tabular}

Fig. 3 Chromaticity of the calibration pattern as a function of Adobe's percent brightness. The mean chromaticity values $(x, y)$ are indicated in the legends

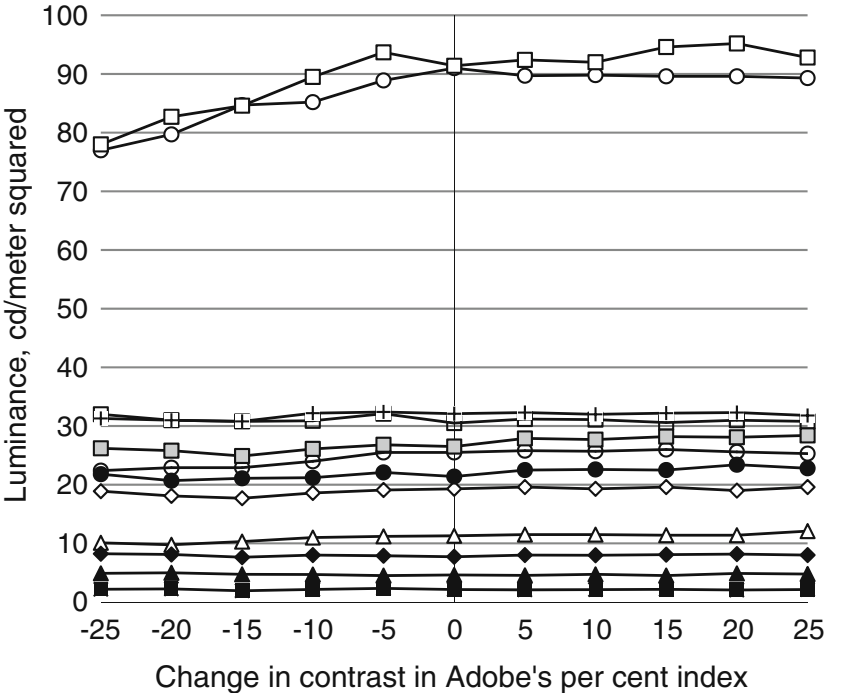

Fig. 4 Luminance of the calibration pattern as a function of Adobe's percent contrast. The slopes of least-square lines are indicated in the legends

In a given trial, each participant first observed the standard and then the comparison, and was instructed not to gaze at the limited area of the picture but to look at the whole of the picture without concentrating too much. In the

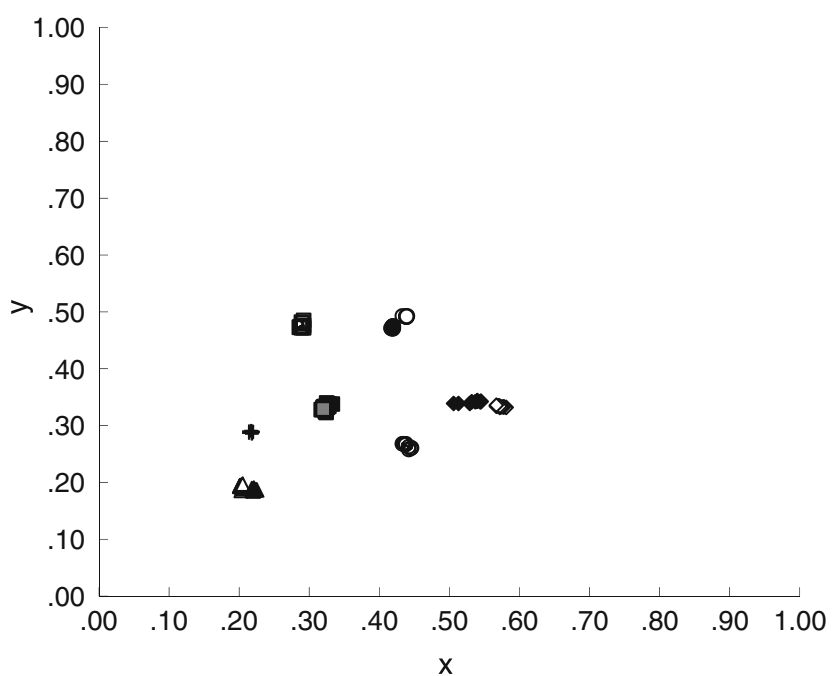

$$
\begin{aligned}
& \neg \operatorname{Red}(.53, .33) \quad \square-G r e e n(.29, .48) \quad-\quad \text { Blue(.20, .19) } \\
& \multimap-\text { Yellow(.44, .49) —-Cyan(.22, .29) - Magenta(.44, .27) } \\
& \rightarrow \square \text { White(.33, .34) } \rightarrow \text { Black(.32, .33) } \quad \square-G r a y(.32, .33) \\
& \rightarrow \text { Dark Red(.53, .34) } \rightarrow \text { Dark Yellow(.42, .47) } \longrightarrow \text { Dark Blue(.22, .19) }
\end{aligned}
$$

Fig. 5 Chromaticity of the calibration pattern as a function of Adobe's percent brightness. The mean chromaticity values $(x, y)$ are indicated in the legends 
brightness judgments, the comparisons that differed in brightness were presented one at time and the participant was required to judge whether the comparison was brighter or darker than the standard. Brightness was defined as visual quality that changes along the dark-dim-bright dimension and seemed to be easily understood by the participant. In image-definition judgments, the comparisons that differed in contrast were presented one at time and the participant was required to judge whether the comparison was more or less distinct than the standard. Image definition was referred to as visual quality that changes in clearness, sharpness, and vividness. For those who appeared to find this explanation of image definition difficult to understand, it was also referred to as the degree to which things in a picture (e.g., people and flowers) are clearly separated from the background.

Each participant made a total of 88 judgments (4 standards $\times 11$ comparisons $\times 2$ conditions). For each standard under the experimental or control conditions, the participant consecutively judged the 11 different comparisons that were presented pseudo-randomly. Each participant was allowed to view the standard whenever he or she wanted to do so. After this block of trials had been completed, the standard was replaced and the condition was alternated. Thus, the experimental conditions and the control conditions were alternated every time the participant completed one block of trials. Each standard was presented twice, one for the experimental condition and the other for the control condition, with the restriction that the order of the standards and the order of conditions for each standard were counterbalanced among participants.

To examine how well the comparisons were perceptually discriminated in brightness, six "raters", who were chosen randomly from the participants of Experiments 1 to 3, were required to place the 11 comparisons for brightness judgments in sequence from the darkest (scored 1) to the brightest (scored 11). The same raters were also required to place the 11 comparisons for image-definition judgments in sequence from the least distinct (scored 1) to the most distinct (scored 11). In the ordering of the comparisons, each rater was allowed to place the comparisons on a desk, compare them simultaneously, and to change the order of the comparisons until he or she was satisfied with the order. Three raters first judged brightness and then image definition, whereas the others judged them in reverse order. The order of the four sets of comparisons for brightness or image-definition judgments was counterbalanced among the raters.

\section{Results}

Ordering of comparisons The upper and lower panels of Fig. 6 show the mean rating of brightness and image definition of the comparisons, respectively. The parameter is the standard. Each data point was averaged across raters. Clearly, the mean rating was nearly a linear function of change in brightness or contrast of the comparison, although the image-definition judgments were more variable than the brightness judgments. A two-way repeated-measure ANOVA was performed on the brightness ratings: the main effect of comparison was significant, $F(10,150)=214.3, p<$ .001 , but the main effect of standard was not significant and the comparison $\times$ standard interaction was not significant. A two-way repeated-measure ANOVA was also performed on the image-definition ratings: the main effect of comparison was significant, $F(10,150)=110.7, p<.001$, but the main effect of standard was not significant and the comparison $\times$ standard interaction was not significant. These results imply that the comparisons were successfully discriminated in brightness and image definition.
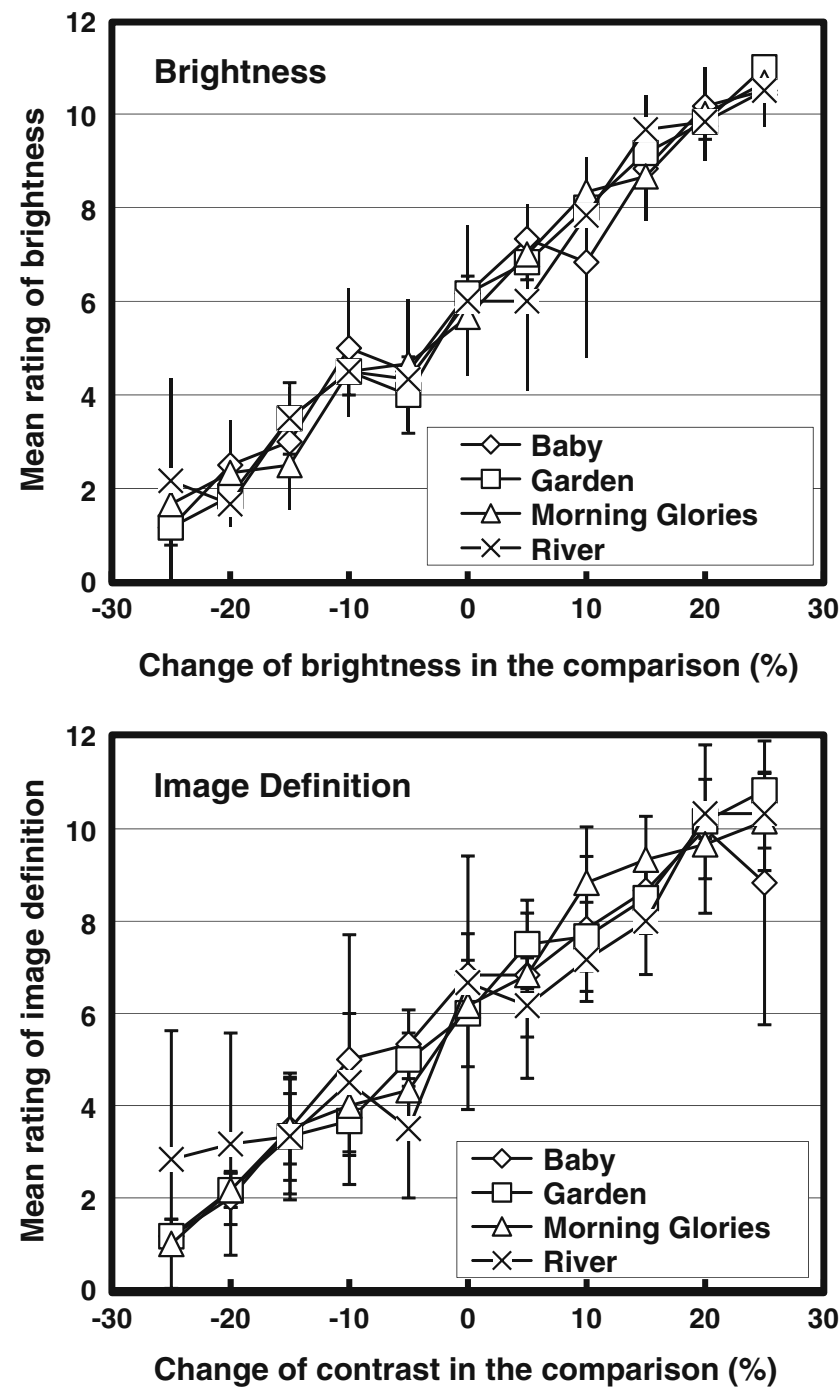

Fig. 6 Mean rating for naturalistic scene pictures as a function of brightness (upper) and of contrast (lower) in the comparison. The parameter is the picture 
Brightness Figure 7 shows the results for the brightness judgments of the comparison relative to the standard. The upper, middle, and bottom panels represent the outcomes for Experiments 1, 2, and 3, respectively. In each panel, the abscissa is the change in Adobe's brightness index of the comparison (\%) and the ordinate is the proportion of "brighter" responses that were averaged across the standards. The parameter is the condition.

A probit analysis was applied to each curve in Fig. 7. For the experimental condition of Experiment 1, the point of subjective equality (PSE) was -2.0 with the $95 \%$ confidence interval (CI) of -4.0 to -0.0 , whereas for the control condition, the PSE was -11.8 with the $95 \%$ CI of -13.8 to -9.9 (the unit of the PSE and its $95 \% \mathrm{CI}$ is per cent). Note that the PSE for the experimental condition did not fall within the $95 \%$ CI for the control condition, and the PSE for the control condition did not fall within the $95 \%$ CI for the experimental condition. Thus, the mean PSE for the experimental condition was significantly different (i.e., brighter) from that for the control condition. If this kind of relationship holds between the experimental and the control conditions in the subsequent experiments, it was decided that the difference in PSE between them is significant.

For Experiment 2, the PSE for the experimental condition was -5.9 with the $95 \%$ CI of -7.4 to -4.5 , whereas the PSE for the control condition was -9.2 with the $95 \%$ CI of -10.7 to -7.9 . Again, the PSE for the experimental condition did not fall within the $95 \%$ CI for the control condition, and the PSE of the control condition did not fall within the $95 \%$ CI of the experimental condition. Thus, the mean PSE for the experimental condition was significantly different from that for the control condition.

For Experiment 3, the PSE for the experimental condition was -8.3 with the $95 \%$ CI of -9.8 to -6.9 ,
Fig. 7 Proportion of "brighter" judgments as a function of change in brightness of the comparison (\%). The upper, middle, and bottom panels are the results for Experiments 1 (inversion of both the retinal image and the head), 2 (inversion of only the head), and 3 (inversion of only the retinal image), respectively
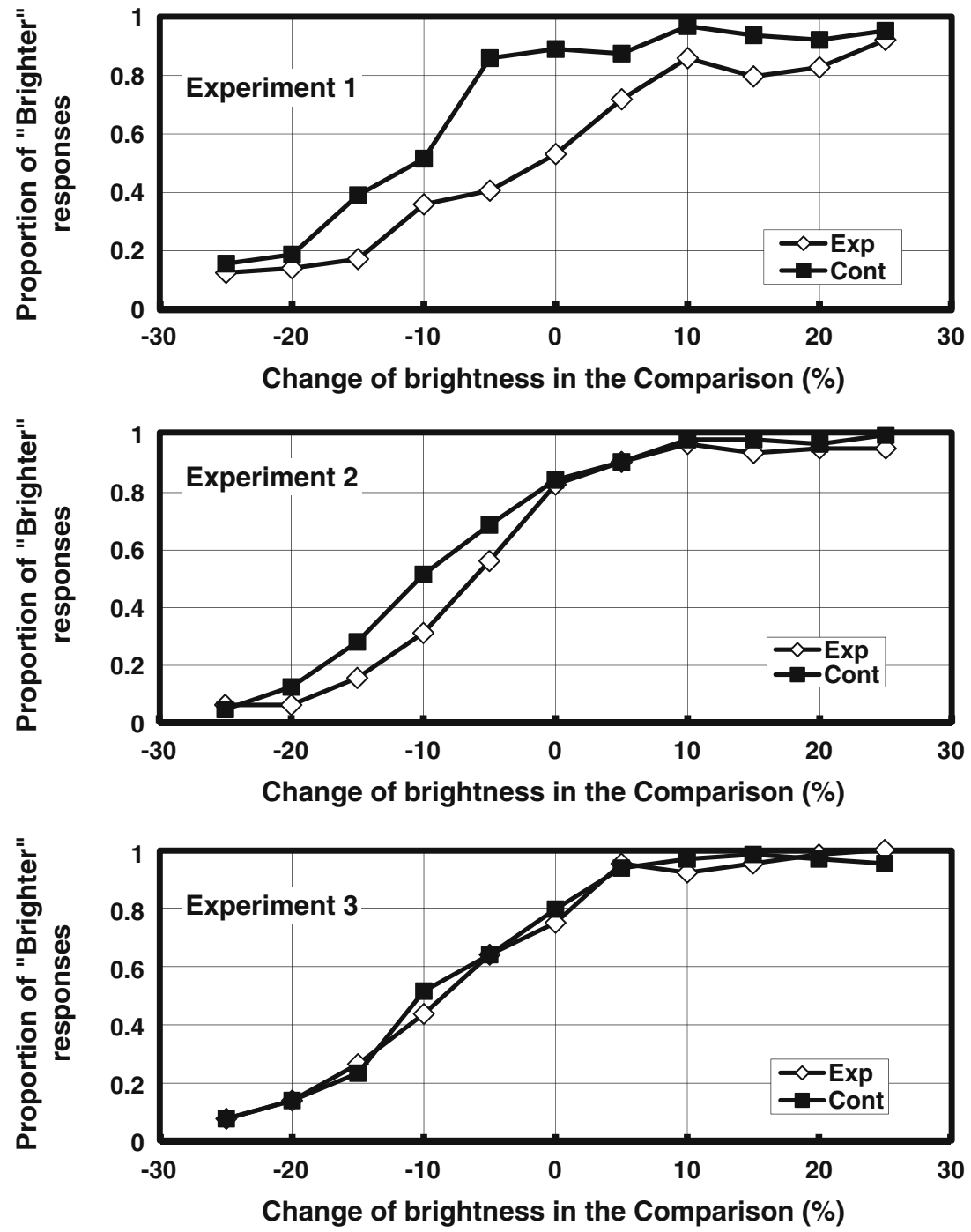
whereas the PSE for the control condition was -8.7 with the $95 \% \mathrm{CI}$ of -10.3 to -7.2 . The PSE for the experimental condition fell within the $95 \%$ CI for the control condition, and the PSE for the control condition fell within the $95 \%$ CI for the experimental condition. Thus, the mean PSE for the experimental condition was not significantly different from that for the control condition.

The PSEs for the experimental condition were compared among Experiments 1, 2, and 3. The PSE for Experiment 1 $(-2.0)$ was significantly different from that for Experiment $2(-5.9)$ and was also significantly different from that for Experiment 3 (-8.7). The PSE for Experiment 2 was significantly different from that for Experiment 3.

Table 1 indicates the PSE and the 95\% CI for each combination of standard and experiment. In Experiment 1, for every standard, there was a significant different in PSE between the conditions. In Experiment 2, for Baby, River, or Garden, there was a significant difference in PSE between the conditions, but for Morning Glories, there was no significant difference between them. In contrast, in Experiment 3, there was no significant difference in PSE for any standard.

Image definition Figure 8 shows the results for imagedefinition judgments of the comparison relative to the standard. The upper, middle, and bottom panels represent the outcomes for Experiments 1, 2, and 3, respectively. In each panel, the abscissa is the change in contrast of the comparison (\%) and the ordinate is the proportion of "more distinct" responses that were averaged across the standards. The parameter is the condition.

For Experiment 1, the PSE for the experimental condition was 8.7 with the $95 \% \mathrm{CI}$ of $7.0-10.5$, whereas the PSE for the control condition was 1.9 with the $95 \%$ CI of $0.4-3.4$. Thus, the mean PSE for the experimental condition was significantly different from that for the control condition.

For Experiment 2, the PSE for the experimental condition was 9.9 with the $95 \%$ CI of $8.6-11.2$, whereas the PSE for the control condition was 4.9 with the $95 \%$ CI of 3.5-6.3. Thus, the mean PSE for the experimental condition was significantly different from that for the control condition.

For Experiment 3, the PSE for the experimental condition was 4.9 with the $95 \%$ CI of 3.7-6.1, whereas the PSE for the control condition was 3.7 with the $95 \%$ CI of 2.5-4.9. Thus, the mean PSE for the experimental condition was not significantly different from that for the control condition.

The PSEs for the experimental conditions were compared among Experiments 1, 2, and 3. The PSE for Experiment 1 (8.7) was not significantly different from that of Experiment 2 (9.9) but was significantly different from that for Experiment 3 (4.9). The PSE for Experiment 2 was significantly different from that for Experiment 3.

Table 2 indicates the PSE and the 95\% CI for each combination of standard and experiment. The results of Experiments 1 and 2 were similar to one another: In either experiment, a difference between the conditions was significant for Baby, Garden, and Morning Glories, but was not significant for River. However, in Experiment 3, a difference between the conditions was significant only for Garden and Morning Glories.

\section{Discussion}

We can be sure that brightness and image definition of the pictures were enhanced by observing them between the legs. The comparison of PSEs between the experimental and control conditions in Experiments 1 to 3 consistently suggests that the change in brightness and image definition was ascribed to the change in proprioception of the head rather than the change in orientation of the retinal image. In addition, the difference in PSE between the inverted-onlyretinal-image condition (Experiment 3) and the full inversion condition (Experiment 1) also suggests that the change in proprioception of the head is critical in both brightness and image-definition judgments.
Table 1 PSE (in \%) and the $95 \%$ confidence interval (in \%) for brightness judgments of the comparison when compared to the standard

*PSE in the experimental condition did not fall within the $95 \% \mathrm{CI}$ in the control condition, and PSE in the control condition did not fall within the $95 \% \mathrm{CI}$ in the experimental condition

\begin{tabular}{lllll}
\hline Condition & Baby & River & Garden & Morning Glories \\
\hline Experiment 1 & & & & \\
Exper. & $-6.6(-12.1 \text { to }-2.0)^{*}$ & $3.8(0.5 \text { to } 7.2)^{*}$ & $-6.4(-10.2 \text { to }-2.8)^{*}$ & $0.4(-3.4 \text { to } 4.6)^{*}$ \\
Control $-15.0(-19.0$ to -11.8$)$ & $-8.9(-11.9$ to -6.0$)$ & $-13.3(-18.7$ to -9.3$)$ & $-10.3(-15.9$ to -6.0$)$ \\
Experiment 2 & & & \\
Exper. & $-5.6(-8.3 \text { to }-3.0)^{*}$ & $-4.0(-7.1 \text { to }-1.0)^{*}$ & $-5.6(-8.6 \text { to }-2.7)^{*}$ & $-8.6(-12.2$ to -5.5$)$ \\
Control & $-12.2(-16.5$ to -8.7$)$ & $-8.8(-11.0$ to -6.6$)$ & $-9.0(-11.8$ to -6.3$)$ & $-8.0(-10.8$ to -5.4$)$ \\
Experiment 3 & & & $-8.8(-9.2$ to -4.4$)$ & $-8.3(-12.4$ to -4.8$)$ \\
Exper. & $-9.9(-13.1$ to -7.0$)$ & $-8.5(-11.5$ to -5.7$)$ & $-6.8 .9(-11.7$ to -4.5$)$ \\
Control & $-10.9(-14.0$ to -8.1$)$ & $-6.7(-9.3$ to -4.2$)$ & $-9.9(-13.6$ to -6.7$)$ & $-7.9(-1)$ \\
\hline
\end{tabular}


Fig. 8 Proportion of "more definite" judgments as a function of change in contrast of the comparison (\%). The upper, middle, and bottom panels are the results for Experiments 1 (inversion of both the retinal image and the head), 2 (inversion of only the head), and 3 ( inversion of only the retinal image), respectively
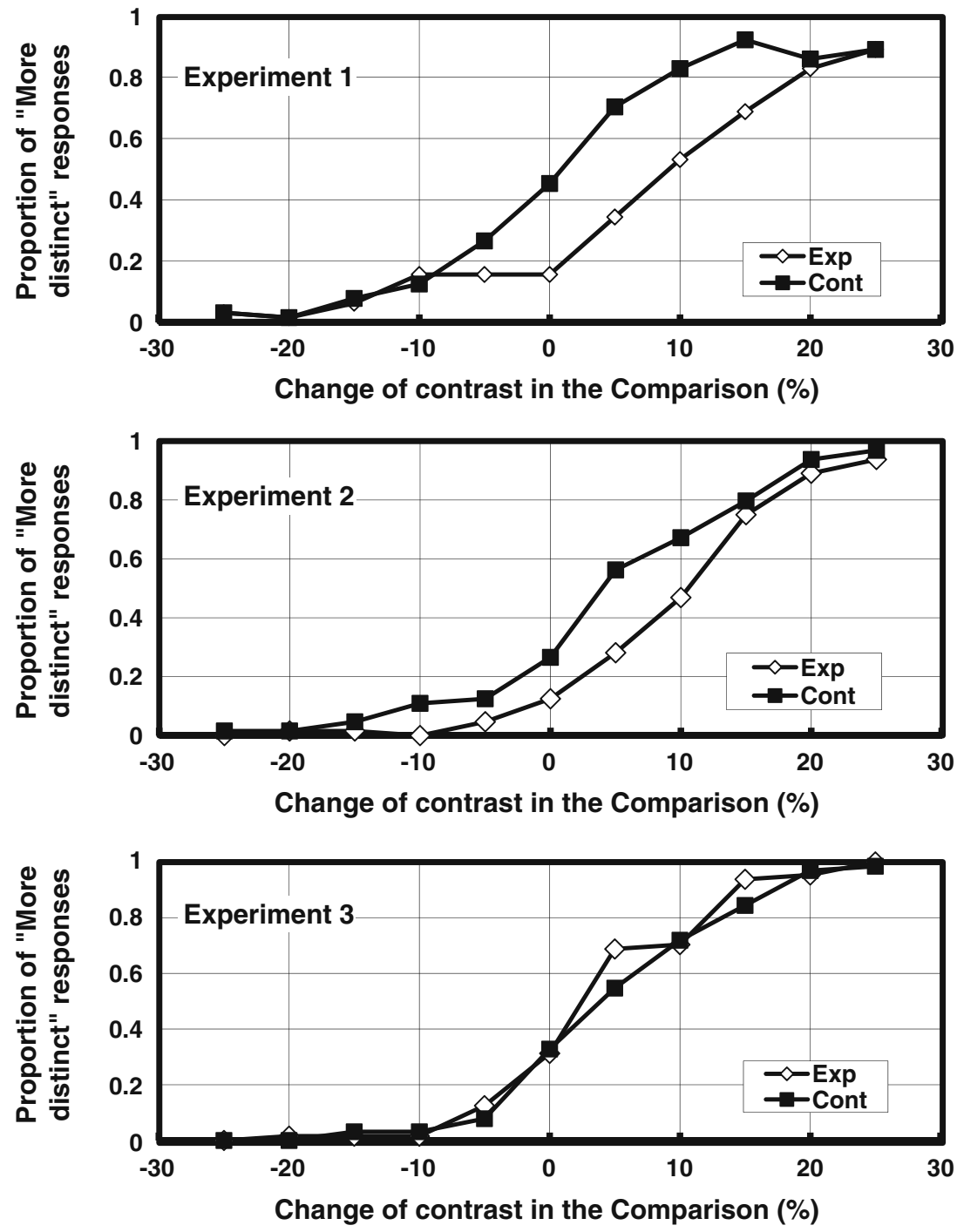

The effect of retinal-image orientation was also found: For brightness judgments, the PSE in the inverted-onlyhead condition (Experiment 2) differed from that in the full inversion condition (Experiment 1). However, this effect was partial because there was not such a difference for image-definition judgments.

\section{Experiment 4}

The results of Experiments 1 to 3 generally suggest that the head-inversion effects are contingent on a change in proprioception of the head, not on a change in orientation of the retinal image. To explain the head-inversion effects,
Table 2 PSE (in \%) and the $95 \%$ confidence interval (in \%) for image-definition judgments of the comparison when compared to the standard

*PSE in the experimental condition did not fall within the $95 \% \mathrm{CI}$ in the control condition, and PSE in the control condition did not fall within the $95 \% \mathrm{CI}$ in the experimental condition

\begin{tabular}{lllll}
\hline Condition & Baby & River & Garden & Morning Glories \\
\hline $\begin{array}{l}\text { Experiment } 1 \\
\text { Exper. }\end{array}$ & $9.1(5.9 \text { to } 12.8)^{*}$ & $5.3(1.1$ to 10.0$)$ & $12.2(9.7 \text { to } 14.8)^{*}$ & $7.9(4.8 \text { to } 11.3)^{*}$ \\
$\begin{array}{l}\text { Control } \\
\text { Experiment } 2\end{array}$ & $3.6(0.8$ to 6.6$)$ & $2.2(-2.3$ to 6.9$)$ & $2.5(-0.3$ to 5.3$)$ & $-0.4(-2.7$ to 2.0$)$ \\
$\begin{array}{l}\text { Exper. } \\
\text { Control }\end{array}$ & $10.4(7.9 \text { to } 13.1)^{*}$ & $9.8(7.1$ to 12.7$)$ & $10.3(8.0 \text { to } 12.5)^{*}$ & $9.2(6.4$ to 12.2$) *$ \\
$\begin{array}{l}\text { Experiment } 3 \\
\text { Exper. }\end{array}$ & $5.1(2.6$ to 7.7$)$ & $7.8(5.0$ to 10.9$)$ & $4.7(1.6$ to 7.9$)$ & $2.2(-0.6$ to 4.9$)$ \\
Control & $1.8(-0.7$ to 4.3$)$ & $4.8(1.9$ to 7.8$)$ & $8.0(5.7 \text { to } 10.3)^{*}$ & $5.3(3.4$ to 7.1$) *$ \\
\hline
\end{tabular}


we can assume that, for a picture seen with the head inverted, apparent depth is reduced and colors are dissociated from objects (i.e., the visual disorder hypothesis). Alternatively, we can assume that a change in proprioception of the head directly affects brightness and image definition (i.e., the proprioception hypothesis). To examine these hypotheses, in Experiment 4, the participants viewed geometric patterns with the head upright or inverted. According to the visual disorder hypothesis, the head-inversion effects would not arise for the geometric patterns, because such patterns do not change their visual appearances even if the orientation of the head is changed. In contrast, if the proprioception hypothesis were correct, the head-inversion effects would arise whenever the head is inverted.

\section{Method}

Participants The participants were 16 university students (5 males and 11 females; age range, 18-23 years; average age, 20.4 years). They were all paid for their participation.

Stimuli The standards were three artificial or naturalistic matte-colored photographic patterns (overlapped circles, overlapped squares, and water waves) as shown in Fig. 1. All standards were geometric patterns that had neither intrinsic orientation nor pictorial cues to depth.

The comparisons for each standard were 11 patterns that were made by Adobe Photoshop, differing from -25 to $+25 \%$ in $5 \%$ steps in brightness or in contrast. The comparisons that differed in brightness were used for brightness judgments and the comparisons that differed in contrast were used for image-definition judgments. All standards and all comparisons were printed on paper by the same printer used in Experiments 1 to 3. All patterns were $21 \mathrm{~cm}$ high $\times 29.5 \mathrm{~cm}$ wide.

Procedure All participants undertook two sessions. Half the participants judged the brightness of the patterns in the first session and judged their image definition in the next session; the remaining participants judged them in the reverse order. Each session contained experimental and control conditions: in the experimental condition, each participant observed the upright standard with the head upside down and then judged the upright comparison with the head upright; in the control condition, each participant observed the upright standard with the head upright and then judged the upright comparison with the head upright. In either condition, each participant was required to say whether the comparison was brighter or darker (or more or less distinct) than the standard.

Each participant made a total of 132 judgments (2 sessions $\times 3$ standards $\times 11$ comparisons $\times 2$ conditions).
For each standard in each session, the participant consecutively judged the 11 different comparisons that were presented pseudo-randomly. The participants were able to see the standards whenever they wanted them during this block of trials. After each block of trials had been completed, the standard was replaced, and the condition was also altered.

To examine how the comparisons were discriminated, six raters, who were chosen randomly from the participants in Experiments 1 to 3, placed the comparisons for brightness judgments in a sequence of brightness and also placed the comparisons for image-definition judgments in a sequence of image definition. This procedure was exactly the same as in Experiments 1 to 3. Three raters first judged brightness and then judged image definition, whereas the others judged them in the reverse order. The order of the three sets of comparisons for brightness or image-definition judgments was counterbalanced among the raters.

\section{Results}

Ordering of comparisons The upper and lower panels of Fig. 9 show the mean rating of brightness and image definition of the comparisons, respectively. The parameter is the standard. Each data point was averaged across raters. The ratings of image definition of the comparisons were more variable than those of brightness.

It seems that the mean rating of brightness increased linearly with change in the brightness of the comparison. A two-way repeated-measure ANOVA was performed on brightness ratings: The main effect of comparison was significant, $F(10,100)=505.0, p<.001$ and the comparison $\times$ standard interaction was significant, $F(20,100)=2.2, p<.01$. This interaction suggested that the mean rating for the $-10 \%$ comparison of Squares was significantly larger than the mean ratings for the $-10 \%$ comparisons of other standards, $F(2,110)=11.9, p<.001$, whereas the mean rating for the $-5 \%$ comparison of Squares was significantly smaller than the mean ratings for the $-5 \%$ comparisons of other standards, $F(2,110)=4.0, p<.05$. Despite this interaction, it seems that for any standard, the comparisons were generally well discriminated in brightness.

A two-way repeated-measure ANOVA was also performed on the image-definition ratings: The main effect of comparison was significant, $F(10,100)=55.9, p<.001$ and the comparison $\times$ standard interaction was significant, $F(20$, $100)=2.2, p<.01$. This interaction suggested that the mean ratings for the 15,20 , and $25 \%$ comparisons of Waves were significantly different from those for the corresponding comparisons of other standards [for the 15 , 20 , and $25 \%$ comparisons, $F(2,110)=23.0,13.3$, and $21.7, p<.001$ for all, respectively]. It seems to be difficult 

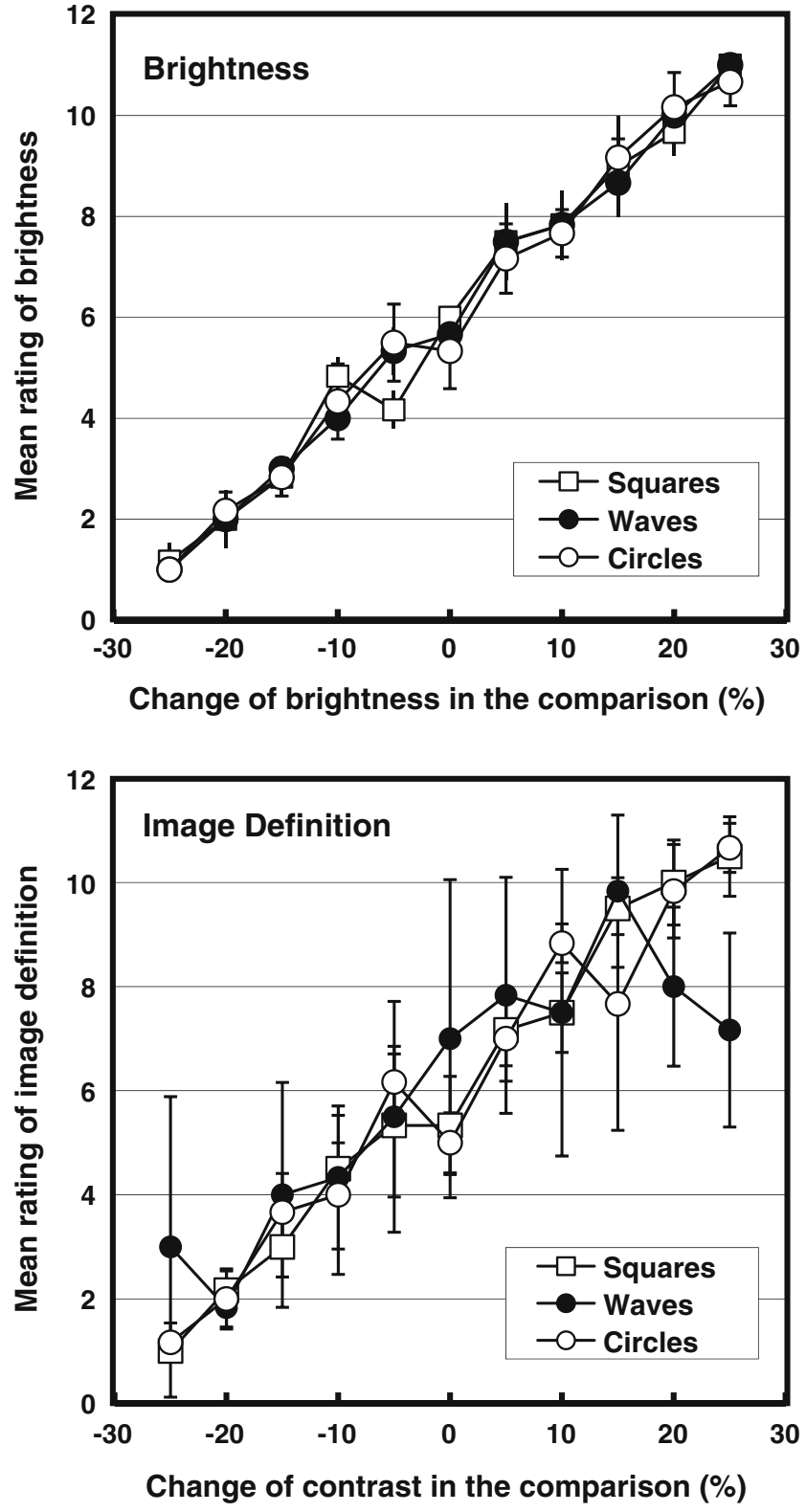

Fig. 9 Mean rating for geometric pictures as a function of change in brightness (upper) and contrast (lower) of the comparison

to discriminate the comparisons of Waves with respect to image definition.

Brightness The upper panel of Fig. 10 shows the results for brightness judgments of the comparison relative to the standard. The abscissa is the change in brightness of the comparison and the ordinate is the proportion of "brighter" responses that were averaged across the standards. The parameter is the condition (the experimental, between-leg viewing; the control, normal upright viewing). It was found that the PSE for the experimental condition was -2.7 with the $95 \%$ CI of -4.4 to -1.0 , whereas the PSE for the control condition was -4.0 with the $95 \%$ CI of -5.5 to -2.6 . This implies that the mean PSE for the experimental condition was not significantly different from that for the control condition. Table 3 indicates the PSE and the 95\% CI for each standard. For every standard, the mean PSE for the experimental condition was not significantly different from that for the control condition.

Image definition The lower panel of Fig. 10 shows the results for image-definition judgments of the comparison relative to the standard. This is based on the results for Circles and Squares, excluding the results for Waves, because we did not obtain the PSE for Waves, which will be explained later. From the lower panel of Fig. 10, the PSE for the experimental condition was -0.2 with the $95 \%$ CI of -2.0 to 1.7 , whereas the PSE for the control condition was -0.9 with the $95 \%$ CI of -3.0 to 1.2 . Thus, the mean PSE for the experimental condition was not significantly different from that for the control condition.
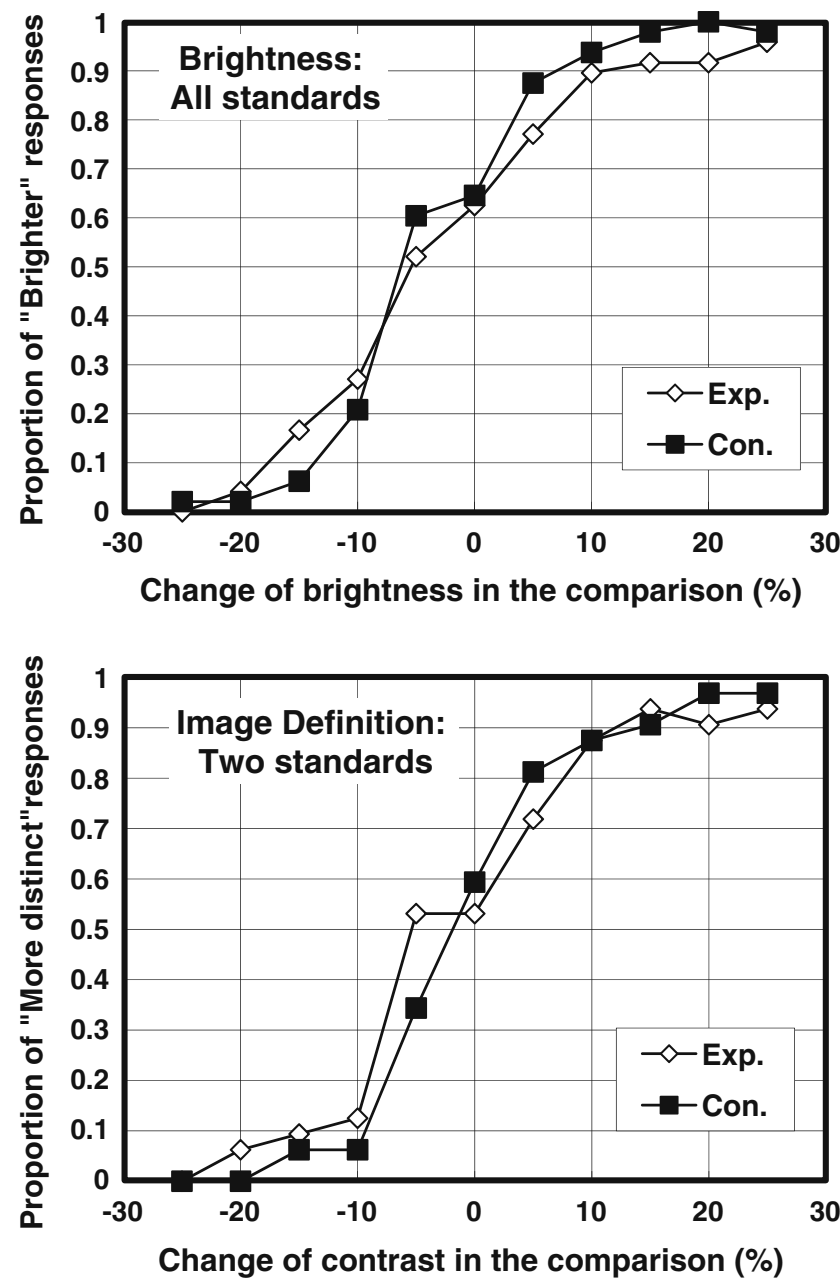

Fig. 10 Proportion of "brighter" (upper) and "more distinct" judgments (lower) as a function of change in brightness and contrast of the comparison (\%), respectively 
Table 3 PSE (in \%) and the $95 \%$ confidence interval (in \%) of the comparisons for brightness and image definition when compared to the standard

\begin{tabular}{llll}
\hline Condition & Circle pattern & Square pattern & Water waves \\
\hline Brightness & & & \\
Exper. & $1.3(-2.1$ to 4.8$)$ & $0.1(-2.6$ to 2.8$)$ & $-9.6(-11.9$ to -7.3$)$ \\
Control & $-3.2(-5.6$ to -0.8$)$ & $-0.4(-2.9$ to 2.1$)$ & $-8.4(-10.8$ to -5.9$)$ \\
Definition & & & \\
Exper. & $-0.3(-4.5$ to 3.9$)$ & $2.5(-3.0$ to 8.5$)$ & Not determined \\
Control & $2.8(-0.9$ to 6.7$)$ & $-2.3(-6.0$ to 1.3$)$ & Not determined \\
\hline
\end{tabular}

Table 3 indicates the PSEs and the 95\% CIs for Circles and Squares. For either standard, the PSE for the experimental condition was not significantly different from that for the control condition. For Waves, the proportion of "more distinct" responses was below chance for nearly all comparisons, and it decreased or remained constant as the contrast of the comparison increased from 0 to $25 \%$.

\section{Discussion}

For brightness judgments, we failed to find the headinversion effects for all geometric patterns: the mean PSE for the between-leg condition did not differ from that for the normal upright condition. For image-definition judgments, the PSEs for Squares and Circles were appropriately determined and indicated that there was no head-inversion effect for these patterns. However, the PSE for Waves was not determined in either condition, because of poor discrimination among the comparisons. Accordingly, in the subsequent discussion, we did not consider the imagedefinition judgments for Waves. The results of Experiment 4, together with the results of Experiments 1 to 3, suggested that, in addition to a change in proprioception of the head, a visual requirement of pictures (i.e., naturalistic scenes with apparent depth and visual orientation) is necessary to produce the head-inversion effects.

\section{Experiment 5}

In Experiments 1 and 4, we found that the head-inversion effects occurred for scene pictures but not for geometric patterns. To explain these results, we can assume that, according to the visual disorder hypothesis, the visual structure of scene pictures is disturbed by way of a change in proprioception of the head. In Experiment 5, to examine this hypothesis, we explored degrees of depth and organization of the pictures that were used in Experiments 1 to 3 . For combinations of retinal-image orientation and head orientation, the participants judged how large the pictures are in the third dimension (i.e., depth judgments) and also judged how well the parts in pictures are related to each other (i.e., organization judgments). If visual disorder of pictures produces a change in brightness and image definition, the visual disorder would occur in the invertedonly-head condition, but not occur in the inverted-onlyretinal-image condition.

\section{Method}

Participants The participants were 16 university students ( 8 males and 8 females; age range, 20-42 years; average age, 28.1 years).

Stimuli The same four standards as in Experiments 1 to 3 were used: Baby, River, Garden, and Morning Glories.

Procedure Each participant was given a preliminary explanation of apparent depth in pictures by observing three dummy pictures with weak, moderate, and strong apparent depth each and choosing the picture with the richest depth and the picture with the poorest depth. The participant was also given an explanation about the relatedness of parts in a picture by observing a well-organized picture (i.e., an upright highway scene composed of the horizontal line, the sky, and the ground) and a poorly-organized picture (an inverted mountain scene covered completely with green trees) and choosing the picture that was easy or difficult to perceive the relationships of its parts.

In the main section of this experiment, Baby, River, Garden, and Morning Glories were presented in a lit room. For the erect position of the head, these pictures were presented on a front wall at eye level, whereas for the bent position, they were presented on a rear wall at eye level. Each participant observed each picture twice under each of four combinations of retinal-image orientation (upright and inverted) and head orientation (upright and inverted). These conditions consisted of the upright pictures seen with the head upright (i.e., the normal upright condition), the inverted pictures seen with the head inverted (i.e., the inverted-onlyhead condition), the inverted pictures seen with the head upright (i.e., the inverted-only-retinal-image condition), and 
the upright pictures seen with the head inverted (i.e., the full inversion condition).

In the first exposure to pictures, half the participants judged a degree of apparent depth of each picture with the five-point scale: no depth (0), weak depth (1), moderate depth (2), strong depth (3), and very strong depth (4), and in the second exposure to the pictures, they judged a degree of organization of each picture with the five-point scale: no organization (0), weak organization (1), moderate organization (2), good organization (3), and very good organization (4). Point " 0 " was assigned to the no-depth or no-organization responses and point "4" was allocated to the very-strong-depth or very-good-organization responses; points " 1, , " 2 ," and " 3 " were successively assigned to alternatives between the extremes. In both the depth and organization judgments, combinations of retinal-image orientation and head orientation were randomly presented to each participant. The other half of the participants first judged organization and then judged apparent depth.

\section{Results}

Depth Figure 11 shows the mean apparent depth for each picture, as a function of combination of retinal-image orientation and head orientation. The vertical attached to each mean indicates the standard deviation. We performed a three-way (retinal image, head, and picture) repeatedmeasure ANOVA on the depth judgments. The main effect of retinal image was significant, $F(1,15)=93.63, p<.001$, suggesting that the mean rating for the inverted retinal image were generally smaller than that for the upright retinal image.

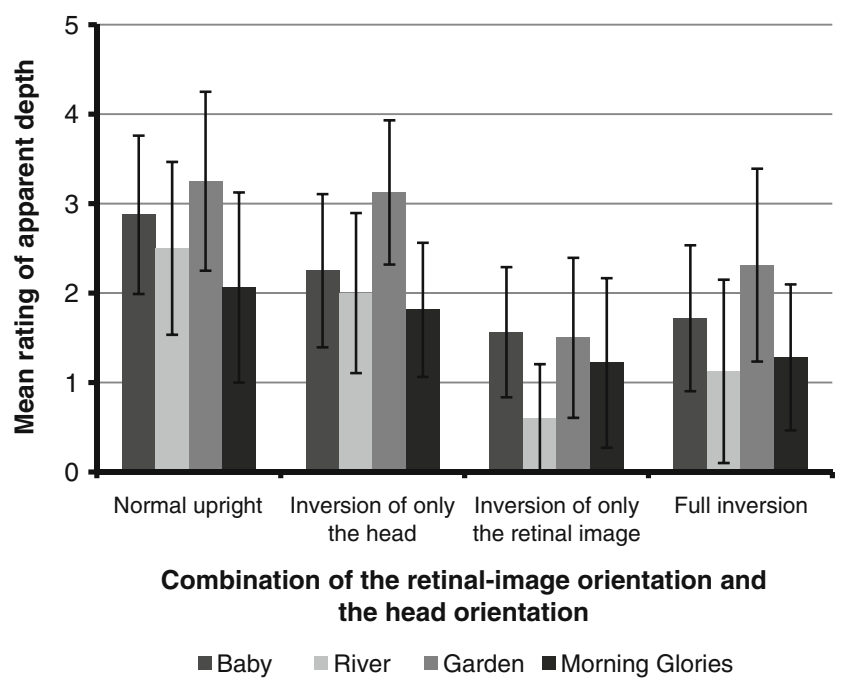

Fig. 11 Mean rating of depth as a function of combination of retinalimage orientation and head orientation. The parameter is the picture
The retinal image $\times$ head interaction was significant, $F$ $(1,15)=16.20, p<.01$. The mean rating for the inverted pictures seen with the head upright (i.e., inverted-onlyretinal-image condition) was significantly smaller than that for the normal upright viewing where both the retinal image and the head were upright $(1.2$ versus 2.7$), F(1,30)=$ $67.57, p<.001$, whereas the mean rating for the inverted pictures seen with the head inverted (i.e., inverted-onlyhead condition) was significantly smaller than the normal upright viewing $(2.3$ vs 2.7$), F(1,30)=5.50, p<.05$. These imply that apparent depth of the pictures was reduced by the change in orientation of the retinal image or by a change in proprioception of the head, and the former was more effective than the latter. Also, the mean rating for the inverted-retinal-image condition was significantly smaller than that for the full inversion condition where both the retinal image and the head were inverted (1.2 vs 1.6$), F(1,30)=$ $4.88, p<.01$, but the mean rating for the inverted-only-head condition was significantly larger than that for the full inversion condition (2.3 vs 1.6$), F(1,30)=15.13, p<.001$. These results also imply that the change in orientation of the retinal image was more effective than the change in proprioception of the head.

The main effect of picture was significant, $F(3,45)=$ $10.57, p<.001$, and the picture $\times$ retinal image interaction was significant, $F(3,45)=4.52, p<.01$. These suggest that in the upright retinal-image conditions, Garden was judged to be the richest in apparent depth, Baby was the richest in the second, River was moderate, and Morning Glories was poor, whereas in the inverted retinal-image conditions, Garden was the richest, Baby was the richest in the second, Morning Glories was moderate, and River was poor.

Organization Figure 12 shows the results of the organization judgments. We performed a three-way repeated-measure ANOVA on the organization judgments. The main effect of retinal image was significant, $F(1,15)=48.56, p<.001$, suggesting that the mean rating for the inverted retinal images was generally smaller than that for the upright retinal images.

The retinal image $\times$ head interaction was significant, $F(1,15)=12.92, p<.01$. The mean rating for the inversion of only the retinal image was significantly smaller than that for the normal upright viewing (1.9 vs 3.2), $F(1,30)=$ $48.26, p<.001$, whereas the mean rating for the inversion of only the head was significantly smaller than for the normal upright viewing (2.7 vs 3.2), $F(1,30)=12.34, p<.01$. Also, the mean rating for inversion of only the retinal image was significantly smaller than that for the full inversion condition (1.9 vs 2.3$), F(1,30)=7.84, p<.01$; whereas the mean rating for inversion of only the head was significantly larger than that for the full inversion condition (2.7 vs 2.3), $F(1,30)=4.93, p<.05$. These results imply that pictures are 


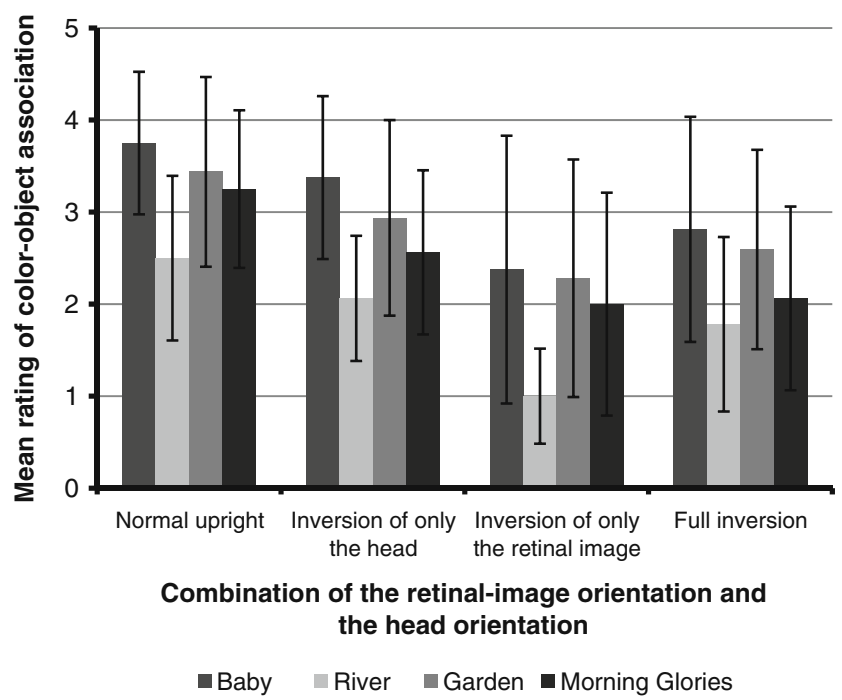

Fig. 12 Mean rating of color-object association as a function of combination of retinal-image orientation and head orientation. The parameter is the picture

perceived to be less organized by the change in orientation of the retinal image or by the change in proprioception of the head, and the former is more effective than the latter.

The main effect of picture was significant, $F(3,45)=$ 9.48, $p<.001$. This suggests that in all conditions, Baby was judged to be best organized, Garden was best organized in the second, Morning Glories was moderately organized, and River was poorly organized.

\section{Discussion}

The following points summarize the results. First, the upright pictures seen with the head upright were judged to be the richest in depth and to be best organized, and the inverted pictures seen with the head upright were judged to be the poorest in depth and to be least organized. This clearly demonstrates that a change in orientation of the retinal image affects apparent depth and organization of pictures. Second, regardless of whether the pictures are upright or inverted, the pictures seen with the head inverted were judged to be poorer in depth and to be less organized than the normal upright viewing. This suggests that a change in proprioception of the head also affects apparent depth and organization of pictures. Third, the change in orientation of the retinal image is more effective than the change in proprioception of the head.

Taken together with the results of Experiments 1 to 3, the present results suggest that for the inverted-only-head condition, visual scenes of the pictures were disorganized and their brightness and definition were enhanced, but for the inverted-only-retinal-image condition, the visual scenes were much more disturbed, but their brightness and image definition remained the same as the normal upright viewing. This delimits the validity of the hypothesis that an increase in brightness and image definition is accompanied by a reduction of apparent depth or dissociation of colors from objects.

\section{General discussion}

We measured brightness and image definition for pictures that were seen with the head upright or inverted. It should be noted that the measurement of image definition seems to be the first attempted in this research field. By these measurements, we have confirmed the head-inversion effects: when scene pictures were viewed with the head upside down, we obtained a $9.8 \%$ increase in brightness and a $6.8 \%$ increase in image definition as compared to normal upright posture. These increases in brightness and image definition were slight but reliable in amount.

More than just confirmation of the effects was obtained in this study. The first is that the head-inversion effects are produced by a change in proprioception that is contingent on bending the head, not by a change in orientation of the retinal image. This means that a proprioceptive factor is a source of the head-inversion effects. This is comparable to Miyakawa's (1949b) results but does not agree with von Helmholtz's note in which a change in orientation of the retinal image is assumed to be critical to the headinversion effects. The second is that the head-inversion effects arise for scene pictures that contain depth cues and intrinsic orientation and do not occur for geometric patterns that appear flat. This means that a visual factor is also a source of head-inversion effects. This is not comparable to Miyakawa's (1949b) results, which showed that the headinversion effects occurred for an achromatic disk as well as a line drawing.

From these findings, we can formulate a rule that changes in brightness and image definition of pictures are obtained if, and only if, naturalistic scene pictures are seen with the head inverted. This rule has two implications. First, if the pictures to be seen are geometric patterns that do not have depth cues and intrinsic orientation, the patterns do not change brightness and image definition even if the head is inverted (Experiment 4). Second, if scene pictures are seen upside down with the head upright, they are poor in apparent depth and are disturbed in colorobject association (Experiment 5) but they do not change in brightness or image definition (Experiment 2). This rule seems to explain all of the results in this study.

However, the first implication mentioned above may be disputable, because there are other possible differences between the scene pictures and the geometric patterns. The 
scene pictures used in this study were made up of plenty of colors, whereas the geometric patterns were made up of only a few colors. If the head-inversion effects may be attributed to this difference in color diversity, it may have nothing to do with apparent depth or visual structure we have for scene pictures. To have access to the possibility of this interpretation, for example, phase-scrambled noise patterns of scene pictures (Phillips \& Todd, 2010; Yoonessi \& Kingdom, 2008) may be compared between the betweenleg condition and the normal upright condition. If the headinversion effects arise in the phase-scrambled noise pattern, where the amplitude spectrum of pictures is reserved but the phase spectrum is scrambled, depth and orientation information is not critical in the head-inversion effects. In the current status of evidence, however, we are not able to rule out either hypothesis, but it is certain that some pictures are more likely to produce the head-inversion effects than others.

The second implication does not support the visual disorder hypothesis that has sometimes been assumed to explain the head-inversion effects (Beck, 1969, 1972; Sanford, 1898; von Helmholtz, 1910/1962). Although scene pictures were certainly disturbed by inverting only the retinal image or by inverting only the head, this disorder did not always connect with a change in brightness or image definition. The largest change in visual structure that is contingent on inversion of only the retinal image did not produce a change in brightness and image definition, as suggested by Beck $(1969,1972)$.

In relation to this, a number of studies (Hochberg \& Beck, 1954; Beck, 1969) have indicated that the perceived lightness of the target is changed by a change in perceived location of a target in depth, without allowing any change in the pattern of light reaching the eyes. A simple demonstration in the classroom is that a bent card, one side of which is illuminated and the other is shaded, changes its lightness when it perceptually flips (Mach, 1886/1959; Goldstein, 1989). The change in lightness in these studies is generally small (i.e., about one Munsell unit), but Gilchrist (1977, 1980) showed that a very drastic change of lightness would be obtained if a target was placed within an ingeniously structured arrangement. The points in which the head-inversion effects differ from these previous studies seem to be that (1) the headinversion effects expand over an entire picture and are not limited to a target in a picture, and (2) the head-inversion effects are affected not only by the visual nature of pictures but also by a change in proprioception of the head.

Mineart (1974/1993) ascribed the head-inversion effects to the greater quantity of blood running to the head when the body was bent forward. If the quantity of blood in the head were a direct determiner of the head-inversion effects, then the effects would arise for the geometric patterns. Furthermore, in literature, we did not find evidence that the quantity of blood running to the head affects perception of brightness and image definition.
Cornish (1935) suggested that, under ordinary conditions of viewing, there is an overestimation of vertical distance as compared with horizontal distance and any unaccustomed posture counteracts this and, by compressing the horizontal bands of color, intensifies their contrast. If this assumption were the case, then the effects would occur for geometric patterns.

In conclusion, we have demonstrated that (1) the headinversion effects are ascribed to a change in proprioception of the head but are not ascribed to a change in orientation of the retinal image, (2) the head-inversion effects are likely to occur for scene pictures but not for geometric patterns, and (3) by inverting only the head or by inverting only the retinal image, the visual structure of pictures was disturbed but the changes in brightness and image definition were limited to the inverted-only-head condition. We also note that there are several problems to be resolved. In particular, we will have to specify visual components in naturalistic scenes that are critical to the head-inversion effects and elucidate how the visual components are integrated with a change in proprioception of the head.

Acknowledgements This study was partially supported by the Ministry of Education, Science, Sports and Culture, Grant-in-Aid for Scientific Research (C), 2008, \#20530672.

\section{References}

Beck, J. (1969). Lightness and orientation. American Journal of Psychology, 82, 359-366.

Beck, J. (1972). Surface color perception (pp. 128-140). Ithaca: Cornell University Press.

Cornish, V. (1935). Scenery and the sense of sight (pp. 15-16). Cambridge: Cambridge University Press.

Galanter, E., \& Galanter, P. (1973). Range estimates of distant visual stimuli. Perception \& Psychophysics, 14, 301-306.

Gibson, J. J. (1950). The perception of the visual world (chapter 3). Boston: Houghton Mifflin.

Gilchrist, A. L. (1977). Perceived lightness depends on perceived spatial arrangement. Science, 195, 185-187.

Gilchrist, A. (1980). When does perceived lightness depend on perceived spatial arrangement? Perception \& Psychophysics, $28,527-538$.

Goldstein, E. B. (1989). Sensation and perception (3rd ed., pp. 179-181). CA: Wadsworth.

Goodale, A. A., \& Milner, A. D. (2004). Sight unseen. Oxford: Oxford University Press.

Higashiyama, A., \& Adachi, K. (2006). Perceived size and perceived distance of targets viewed from between the legs. Vision Research, 46, 3961-3976.

Hochberg, J., \& Beck, J. (1954). Apparent spatial arrangement and perceived brightness. Journal of Experimental Psychology, 47, 263-266.

Howard, I. P. (1982). Human visual orientation. Toronto: Wiley.

Howard, I. P., \& Templeton, W. B. (1966). Human spatial orientation. London: Wiley. 
Mach, E. (1886/1959). In S. Waterlow (Ed.), Analysis of sensation (5th ed., Chapter 10). New York: Dover.

Mineart, M. G. J. (1974/1993). In L. Seymour (Ed.), Light and color on the outdoors (p. 134). New York: Springer.

Miyakawa, T. (1943). Experimental research on the structure of visual space when we bend forward and look backward between the spread legs, I. Japanese Journal of Psychology, 18, 289-309 (in Japanese).

Miyakawa, T. (1949a). Experimental research on the structure of visual space when we bend forward and look backward between the spread legs, II. Japanese Journal of Psychology, 20(2), 14-23 (in Japanese with English abstract).

Miyakawa, T. (1949b). On the change of brightness and color when we bend forward and look backward between the spread legs. Japanese Journal of Psychology, 20(3), 1-5 (in Japanese with English abstract).
Phillips, F., \& Todd, J. T. (2010). Texture discrimination based on global feature alignments. Journal of Vision, 10(6):6, 1-14. doi:10.1167/ 10.6 .6

Ross, H. E. (1974). Behaviour and perception in strange environments. London: Allen and Unwin.

Ross, H. E., \& Plug, C. (2002). The mystery of the moon illusion: Exploring size perception. Oxford: Oxford University Press.

Sanford, E. C. (1898). A course in experimental psychology. Part 1 (pp. 210-212). Boston: Heath.

von Helmholtz, H. (1910/1962). In J. P. C. Southall (Ed.), Helmholtz's treatise on physiological optics (3rd ed., Vol. 3, p. 9). NY: Dover Publications.

Yoonessi, A., \& Kingdom, F. A. A. (2008). Comparison of sensitivity to color changes in natural and phase-scrambled scenes. Journal of the Optical Society of America A, 25, 676-684. 